\title{
Exposition au harcèlement psychologique au travail : Impact sur la satisfaction au travail, l'implication organisationnelle et l'intention de départ
}

\section{Exposure to bullying at work: Impact of job satisfaction, organizational commitment and intention to leave}

\author{
Gwénaëlle Poilpot-Rocaboy*, Guy Notelaers** \& Lars Johan Hauge*** \\ * IGR-IAE de l’Université de Rennes, 11 rue Jean Macé CS 70803, 35708 Rennes Cedex 7, France, \\ gwenaelle.poilpot@univ-rennes1.fr \\ ** Maastricht University, School for Business and Economics, Departement Organization and Strategy, The \\ Netherlands - g.notelaers@maastrichtuniversity.nl \\ *** University of Bergen, Department of Psychology, Bergen Bullying Research Group, Norway
}

\section{Résumé:}

Cet article s'interroge sur les réponses individuelles face à un comportement de harcèlement psychologique. Un modèle conceptuel est proposé et testé sur une population de 6175 personnes, à l'aide d'une approche confirmatoire des modèles d'équations structurelles. Les résultats permettent d'obtenir deux apports essentiels. Le premier est de révéler un effet direct de l'exposition au harcèlement psychologique sur la satisfaction au travail, l'implication organisationnelle et l'intention de départ de la personne exposée. Le second est de révéler le rôle médiateur de la satisfaction au travail entre le harcèlement d'une part et l'implication au travail et l'intention de départ d'autre part. Un processus dominant semble se développer en trois étapes : une insatisfaction au travail comme réponse émotionnelle à l'exposition au harcèlement (première étape), qui se traduit par une réduction de l'implication organisationnelle (seconde étape) et par une augmentation des intentions de départ (troisième étape).

\section{Abstract:}

In this article we investigate the individual reactions to workplace bullying exposure. To this end we put forward a conceptual model which is tested on a heterogeneous sample of 6175 employees using a strict confirmatory approach of structural equation modelling. The results are twofold. Firstly, workplace bullying leads directly to a decrease in job satisfaction and organisational commitment and to an increase in turnover intentions. Secondly, job satisfaction seems to be a partial mediator between workplace bullying on the one hand and organisational commitment and turnover on the other hand. The dominant process may have 3 phases: lowering job satisfaction as emotional reaction to exposure (1) which is translated into lowering organizational commitment (2) and in turn into increasing turnover intentions.

Mots clés: Harcèlement psychologique au travail, satisfaction au travail, implication organisationnelle, intention de départ.

Key words: Bullying, job satisfaction, organizational commitment, intentions to leave. 


\section{Introduction}

Parce que différentes études montrent que le harcèlement psychologique représente une grande menace pour beaucoup de salariés, ce thème a connu un intérêt considérable et croissant dans le monde et est devenu un nouvel objet de recherche en Europe, Australie, Afrique du Sud et Etats-Unis (Einarsen \& Nielsen, 2004 ; Zapf \& Einarsen, 2003). Selon diverses études, plus de 8\% des salariés sont victimes de harcèlement au travail (Nielsen, 2009) et $80 \%$ des lieux de travail seraient concernés par ce risque (Nordic Meeting, 2009).

Le harcèlement psychologique au travail, aujourd'hui légalement condamné en France et en Belgique, correspond à toute attitude durable et répétée d'un ou plusieurs membres de l'organisation qui tend à intimider, à dévaloriser ou à isoler un autre de ses membres ayant pour conséquence de le déstabiliser. La déstabilisation de la personne peut résulter de motivations diverses telles que la distraction, le refus d'une différence, la volonté de nuire, la jalousie, le souhait de conquérir ou de garder le pouvoir, le bénéfice de faveurs à caractère sexuel, l'obtention de la démission (Poilpot-Rocaboy, 1998) ou de la maladresse ou de l'incompétence d'un individu. Ainsi, le harcèlement psychologique apparaît comme une situation dans laquelle un individu est exposé sur une longue période à des agissements répétés et non souhaités, de la part de son supérieur hiérarchique ou de son collègue, créant un environnement psychologique de travail déplaisant lié à l'humiliation, la rancœur et la détresse de la victime (Einarsen, Hoel, Zapf \& Cooper, 2003).

Le harcèlement est un processus dynamique en quatre étapes (PoilpotRocaboy, 2006). L'interaction de trois types d'antécédents -la rencontre de deux ou plusieurs individus (caractéristiques de l'agressé et de l'agresseur) dans un contexte de travail donné (caractéristiques de l'organisation)- (étape 1) fait émerger le comportement de harcèlement psychologique (étape 2). Ce comportement génère des réponses diverses, d'une part, des individus (peur ou incompréhension de la situation par l'agressé ; recherche d'aide auprès des collègues, du DRH, des syndicats, du médecin du travail ; plaintes et recours judiciaire; développement de l'insatisfaction; désengagement vis-à-vis de l'entreprise; intention de quitter l'entreprise...), et, d'autre part, de l'organisation (aucune réaction; recherche d'information pour comprendre la situation; enquête; sanction de l'agresseur...) (étape 3). Ces réponses individuelles et organisationnelles au comportement de harcèlement engendrent des conséquences de trois types (étape 4): 1- conséquences individuelles (dégradation de la santé physique et mentale, dégradation de la situation financière de l'agressé..); 2- conséquences organisationnelles (perte de performance du fait de la non satisfaction de l'agressé et des témoins de la situation de harcèlement, du désengagement et de la non implication de l'agressé et des témoins de la situation de harcèlement, coût lié au départ de salariés et à leur remplacement...) ; 3- conséquences sociétales (coûts générés par la sortie prématurée de salariés; coût des régimes sociaux (prestations 
d'assurance maladie, invalidité, prestations de chômage..); perte de compétences pour le marché du travail du fait d'inaptitudes au travail de longue durée...).

Différents auteurs se sont intéressés à ce processus. Certains tentent de le modéliser d'une manière globale (Ballien, Neyens, De Witte \& Decuyper, 2009 ; Bowling \& Beehr, 2006 ; Einarsen, 1996 ; Poilpot-Rocaboy 2006 ; Zapf, 1999). D'autres analysent une partie du processus tels que ses déterminants (Hauge, Skogstad \& Einarsen, 2007 ; Hodson, Roscigno \& Lopez 2006 ; Hoel \& Salin 2003 ; Notelaers, De Witte \& Einarsen, 2010 ; Zapf \& Einarsen 2003) ou ses conséquences sur le stress et le bien-être au travail (Djurkovic, McCormack \& Casimir 2004; Matthiesen \& Einarsen 2004; McCarthy, Sheehan, \& Kearns 1995; McCarthy, Sheehan, Wilkie \& Wilkie 1998; McCarthy, Rylance, Bennett, Zimmerman 2001) ou sur la performance (Ayoko, Callan \& Hartel 2003; Hoel, Einarsen \& Cooper 2003). D'autres enfin questionnent l'émergence de la notion de harcèlement psychologique au travail (Faulx, 2009) dans les sociétés occidentales depuis une quinzaine d'années.

Notre objectif est d'analyser la phase 3 du processus de harcèlement et de nous interroger sur les réponses individuelles face à un comportement de harcèlement psychologique: Quelles réponses la personne, exposée au harcèlement psychologique, développe-t-elle dans l'organisation?

Nous souhaitons ici analyser trois types de réponses en distinguant la satisfaction au travail que nous qualifions de réponse de nature émotionnelle ${ }^{2}$ et celles de nature comportementale telle que l'intention de départ de l'entreprise ou l'implication au travail. Sur la base de travaux précédents, un modèle théorique est proposé.

\section{Hypothèses de la recherche et modèle conceptuel}

\subsection{Hypothèses de la recherche}

Notre première hypothèse est l'existence d'un impact direct de l'exposition à des agissements de harcèlement psychologique sur la satisfaction au travail.

Locke (1976) définit la satisfaction au travail comme «un état émotionnel positif ou plaisant résultant de l'évaluation faite par une personne de son travail ou de ses expériences de travail ». Elle est ainsi une réponse affective et émotionnelle d'une personne face à une situation de travail qui résulte de l'adéquation entre ce que la personne désire (ses attentes) et ce qu'elle retire de son travail. Hulin et Judge (2003) dans leur revue de littérature

2 La satisfaction se définit comme le contentement, le plaisir qui résulte de l'accomplissement de ce qu'on attend, de ce qu'on désire (Larousse) 
sur la satisfaction au travail confirment cette dimension émotionnelle et définissent le concept comme «...des réponses émotionnelles multidimensionnelles à un travail. Ces réponses ont des composantes cognitives, affectives et comportementales. La satisfaction se réfère à des états cognitifs et affectifs internes...»(p.255-256).

Notre hypothèse est qu'une situation de harcèlement est subie par l'individu qui en est la cible. De ce fait, une telle expérience génère un état émotionnel négatif et donc de l'insatisfaction au travail. Pour aller dans ce sens, Quine (1999), Bowling et Beehr (2006) mettent en exergue un lien de corrélation négatif entre le harcèlement psychologique et la satisfaction au travail. Djurkovic, McCormack et Casimir (2004) montrent de même que le harcèlement psychologique développe des affects négatifs au travail.

\section{Hypothèse 1: L'exposition à des comportements de harcèlement psychologique est négativement corrélée à la satisfaction au travail de la personne exposée}

Notre seconde hypothèse est celle de l'existence d'un impact de l'exposition à des agissements de harcèlement psychologique sur l'implication organisationnelle.

Dans un premier temps, un impact direct est attendu. Mowday, Porter et Steers (1982) dénombrent une trentaine de définitions de l'implication organisationnelle et la caractérisent par une forte croyance dans les buts et les valeurs de l'organisation, par une volonté d'exercer des efforts significatifs au profit de celle-ci et au fort désir d'en rester membre. Mowday (1998) complète ses travaux et définit l'implication organisationnelle comme une force générale conduisant l'individu à s'identifier et à s'engager envers l'organisation dans laquelle il travaille.

Il nous semble difficile de concevoir qu'un individu puisse s'identifier à une organisation qui harcèle. De ce fait, notre hypothèse est que le harcèlement limite l'implication organisationnelle. Cette hypothèse se justifie notamment par les travaux sur le contrat psychologique (Levinson, Price, Munden, Mandl et Solley, 1962 ; Schein, 1965). Le contrat psychologique est défini comme la résultante de la perception des obligations réciproques des individus et de l'organisation dans la relation de travail. Ce contrat recense l'ensemble des perceptions de ce que chaque partie est en droit de recevoir et a l'obligation de donner en échange de la contribution de l'autre partie. Ces perceptions sont basées sur des promesses perçues individuellement et ne sont pas nécessairement reconnues par tous les acteurs de l'organisation. Dans des travaux précédents, le respect mutuel est apparu comme l'un des termes de l'échange dans le cadre du contrat psychologique relationnel (Poilpot-Rocaboy, 1998). Le non respect de la personne humaine en situation de travail, illustré par 
l'exposition au harcèlement psychologique engendre ainsi le déséquilibre du contrat qui conduit à un réajustement des comportements de la personne exposée. Ce réajustement peut se traduire notamment par un désengagement de cette personne. Bowling et Beehr (2006) confirment cette hypothèse et mettent en avant une corrélation négative directe entre le harcèlement et l'implication dans l'organisation.

\section{Hypothèse 2a : L'exposition à des comportements de harcèlement psychologique limite directement l'implication organisationnelle de la personne exposée}

Dans un second temps un impact indirect du harcèlement sur l'implication au travail est attendu. La représentation unidimensionnelle de l'implication proposée par Mowday et al. (1982) côtoie une représentation tridimensionnelle (Meyer et Allen, 1997) où l'implication organisationnelle résulte d'un triple engagement affectif, raisonné et moral. Sur la base de cette perception, Durrieu et Roussel (2002) définissent l'implication organisationnelle comme «l'attitude de l'individu correspondant d'une part à son attachement affectif à l'organisation dans laquelle il partage des valeurs communes; d'autre part à son choix raisonné de lui rester fidèle, fonction de l'évaluation qu'il fait du coût d'opportunité de partir ou de rester, enfin à l'obligation morale qu'il ressent de lui rester fidèle et d'accomplir son devoir jusqu'au bout ».

Cette définition nous conduit à supposer que l'attachement affectif est associé à l'état émotionnel d'une personne. De ce fait, la satisfaction au travail peut être perçue comme une variable médiatrice entre l'exposition à un comportement de harcèlement psychologique et l'implication organisationnelle de la personne. Ainsi, l'état émotionnel négatif de la personne exposée la conduit à une attitude de désengagement au sein de l'entreprise. McCormack, Casimir, Djurkovic et Yang (2006) mettent en avant un lien de corrélation entre le harcèlement psychologique, la satisfaction au travail et l'implication organisationnelle et montrent que la baisse de satisfaction liée à l'exposition au harcèlement est corrélée à une baisse d'implication organisationnelle.

\section{Hypothèse 2b : L'exposition à des comportements de harcèlement psychologique engendre un état émotionnel négatif qui limite l'implication organisationnelle de la personne exposée}

Notre troisième hypothèse est celle d'un impact de l'exposition à des agissements de harcèlement psychologique sur l'intention de départ de la personne cible. L'intention de départ met en avant l'intention qu'a un salarié de remettre en cause son adhésion comme membre au sein de l'organisation. Elle 
implique l'évaluation de l'utilité à quitter l'entreprise par opposition à celle de rester (Mueller, Wallace \& Price, 1992).

Dans un premier temps, l'hypothèse d'un impact direct du harcèlement sur l'intention de départ de la cible du harcèlement est proposée. Différents travaux vont dans le sens de la validation de cette hypothèse. Rayner et Cooper (1997) dans une étude menée au Royaume-Uni montrent que $27 \%$ des personnes harcelées répondent à cette situation en quittant leur emploi. Zapf et Gross (2001) évoquent que les stratégies de réponse des victimes évoluent dans le temps. Les victimes semblent utiliser des stratégies de résolution de conflits au début du processus, puis changent de stratégies plusieurs fois et finissent, pour certains, par tenter de quitter l'entreprise. Dans une méta-analyse, Bowling et Beehr (2006) mettent en avant une corrélation positive entre le harcèlement et l'intention de quitter l'entreprise. Cette hypothèse, comme l'hypothèse $\mathrm{H} 2 \mathrm{a}$, se justifie par les travaux sur le contrat psychologique (Levinson, Price, Munden, Mandl et Solley, 1962 ; Poilpot-Rocaboy, 1998 ; Schein, 1965). Le non respect de la personne humaine en situation de travail, illustré par l'exposition au harcèlement psychologique, engendre le déséquilibre du contrat psychologique relationnel qui conduit l'individu à le remettre en cause en quittant l'organisation. Notelaers et De Witte (2003) confirment cette hypothèse d'impact de l'exposition à des comportements de harcèlement sur l'intention de départ de l'individu.

\section{Hypothèse 3a: L'exposition à des comportements de harcèlement psychologique impacte directement l'intention de départ de l'entreprise de la personne exposée}

Dans un second temps, il peut être supposé que la satisfaction au travail est aussi une variable médiatrice entre l'exposition aux comportements de harcèlement psychologique et l'intention de départ de l'entreprise. Ainsi, l'état émotionnel négatif de la personne exposée la conduit à envisager à quitter l'organisation.

Divers travaux ont mis en exergue la relation entre insatisfaction au travail et intention de départ. Par exemple, Lambert, Hogan et Barton (2001) mettent en avant le rôle médiateur de la satisfaction au travail entre l'environnement de travail et l'intention de départ de l'entreprise. Crede, Chernyshenko, Stark, Dahal et Bashshur (2007) montrent que la satisfaction au travail est une variable médiatrice de la relation entre des variables contextuelles internes et externes (facteurs économiques, caractéristiques de l'emploi, réactions à des évènements dans l'entreprise (stress, injustice...), caractéristiques de l'individu (extraversion, affects positifs ou négatifs...) et trois types de comportements au travail : 1- désengagement au travail (intention de départ, retard...), 2- comportements dysfonctionnels (dégradation de matériel, vol, mauvaise qualité du travail...), 3- comportements positifs (aide 
les autres, suggestion d'amélioration au sein de l'organisation, respect et défense d'actions «morales» dans l'entreprise...). Plus précisément, des travaux mettent en avant un lien entre l'exposition à des comportements de harcèlement, la satisfaction au travail et l'intention de départ de l'individu. Djurkovic, McCormack et Casimir (2004) montrent que le développement d'affects négatifs engendré par le harcèlement psychologique conduit les personnes visées à quitter l'entreprise.

\section{Hypothèse 3b : L'exposition à des comportements de harcèlement psychologique engendre une absence de satisfaction qui génère l'intention de départ de l'entreprise de la personne exposée}

Enfin, des travaux ont montré le lien entre l'implication organisationnelle et l'intention de départ. Maertz et Campion (1998) font une synthèse de ces travaux et montrent que la satisfaction au travail et l'implication organisationnelle sont des déterminants de l'intention de départ. Mowday et al. (1982), Mueller et al. (1992) ainsi que Griffeth, Hom et Gaertner (2000) confirment et considèrent la satisfaction au travail et l'implication organisationnelle comme des variables explicatives de l'intention de départ. De même, Tett et Meyer (1993) dans une méta-analyse concluent que la satisfaction et l'implication contribuent indépendamment à la prédiction de l'intention de départ. Meyer et al. (2002) confirment que l'implication est négativement corrélée à l'intention de départ. Nous souhaitons ici tester l'existence d'un lien direct entre l'implication organisationnelle et l'intention de départ de l'entreprise. La perte d'implication organisationnelle engendrée par l'exposition à des agissements de harcèlement psychologique au travail accroît l'intention de départ de l'entreprise (hypothèse 4).

\section{Hypothèse 4: L'implication organisationnelle est négativement corrélée à l'intention de quitter l'entreprise}

\subsection{Le modèle testé}

Sur la base des hypothèses ci-dessus, un modèle conceptuel est proposé dans la figure 1. Il met en relation le concept de harcèlement psychologique au travail et les stratégies de réponse des personnes qui y sont exposées. Le comportement de harcèlement psychologique est perçu comme générateur d'émotions négatives qui impactent la satisfaction au travail, l'implication organisationnelle et l'intention de départ de l'entreprise. Ainsi, cette relation entre le harcèlement et les réponses individuelles développées pour y faire face, apparait comme une relation complexe où se mêlent des émotions négatives génératrices d'attitudes et de comportements (décisions d'action). 
Figure 1: Stratégies de réponse des personnes exposées au harcèlement psychologique : Le modèle testé

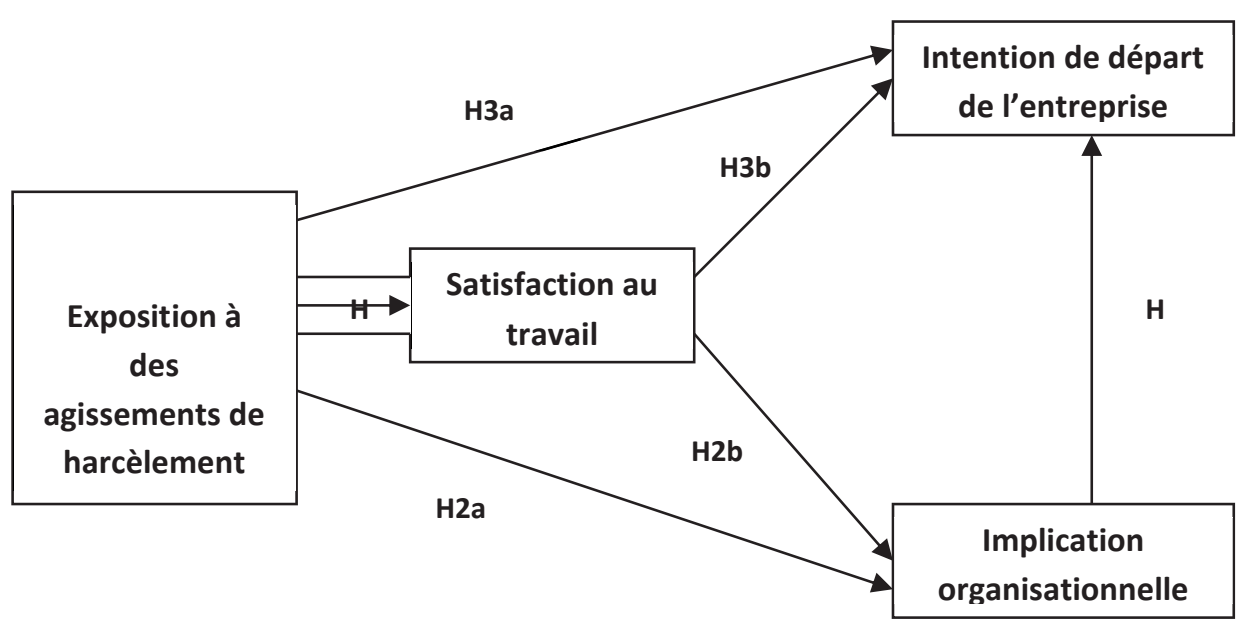

\section{Méthodologie}

\subsection{L'échantillon}

Environ 9000 questionnaires ont été administrés en Belgique par le premier auteur en collaboration avec des agences de santé et des étudiants faisant leur thèse de master. Les questionnaires avaient pour but d'évaluer le bien-être des individus au travail en général et incluaient les thématiques plus précises de harcèlement psychologique et de stress au travail. Une fois rempli, les questionnaires étaient remis dans une urne ou envoyés directement aux agences ou au premier auteur.

6175 participants de 18 organisations belges ont répondu soit un taux de réponse de près de $69 \% .57 \%$ des personnes ont répondu à un questionnaire écrit en néerlandais et $43 \%$ à un questionnaire écrit en français. La moyenne d'âge des répondants est de 41 ans. L'échantillon est composé de 52\% d'hommes. Le secteur d'activité des personnes ayant répondu au questionnaire est pour $48 \%$ le secteur privé, pour $27 \%$ le secteur public de la santé et $25 \%$ les institutions gouvernementales. La répartition par groupes professionnels est: $9 \%$ d'ouvriers, $31 \%$ d'agents de maitrise, $4 \%$ de travailleurs sociaux, $13 \%$ d'infirmières, $21 \%$ fonctionnaires, $10 \%$ de cadres moyens et $11 \%$ de cadres supérieurs. $78 \%$ des répondants ont un contrat à durée indéterminée (CDI), 15\% 
ont un contrat à durée déterminée (CDD) et $7 \%$ ont un autre type de contrat (intérim, sous-traitant ou indépendant).

\subsection{La mesure des concepts}

\subsubsection{Le harcèlement psychologique au travail}

Deux types de mesures peuvent être mobilisés pour évaluer le harcèlement psychologique au travail, l'un dit subjectif et l'autre dit objectif (Zapf \& Einarsen, 2003). La méthode subjective de « self-labelling » consiste à opposer une population harcelée à une population non harcelée, en questionnant directement une personne sur le fait qu'elle considère ou non être harcelée (après éventuellement avoir défini le harcèlement). La méthode objective tend à évaluer l'exposition au harcèlement à l'aide d'une liste d'agissements, la personne devant préciser si elle subit ou non chacun des agissements proposés. Cette méthode se focalise sur la description des comportements subis et ne demande pas directement à la personne si elle est victime de harcèlement. Dans le cadre de la méthode objective, différents outils de mesure sont utilisés dans la littérature mais le questionnaire «NAQ» (Negative Act Questionnaire) (Einarsen \& Hoel, 2001; Einarsen \& Raknes 1997) tend à s'imposer. En effet, les tests effectués semblent garantir sa validité prédictive et de construit et assurer une mesure fiable du harcèlement psychologique au travail (Einarsen, Hoel \& Notelaers, 2009).

Dans cette recherche, l'exposition au harcèlement a été mesurée par une version courte du « Negative Act Questionnaire » (NAQ) traduit en néerlandais et en français par Notelaers (Notelaers, et al. 2006) et développée par Notelaers et Einarsen (2008). Cette version du questionnaire comprend neuf items : Quelqu'un retient de l'information qui vous est nécessaire et rend ainsi votre travail difficile; Rumeurs à votre encontre ; Exclusion des activités de groupe, exclusion de la part des collègues ; Remarques répétées à l'encontre de votre vie privée ou de vous-même; Insultes; Remarques répétées concernant vos erreurs ; Silence ou hostilité face à vos questions ou vos tentatives d'entrer en conversation; Dévalorisation de votre travail et de vos efforts ; «Blagues », « surprises » désagréables. Pour chacun des neuf comportements, la question est « Durant les six derniers mois, à quelle fréquence avez-vous été sujets aux actes suivants? ». L'échelle de mesure des neuf items dans la version du «NAQ » utilisée ici est : « jamais », « de temps en temps », " une fois par mois », « une fois par semaine ou plus ». Cette version a démontré une validité prédictive supérieure à celle du questionnaire initial. En effet, l'homogénéité de l'instrument de mesure est satisfaisante car la consistance interne évaluée par le Alpha de Cronbach est de 0.79. A l'exception du premier item dont la contribution factorielle n'est que de 0.51 , les autres items atteignent un niveau situé entre 0.75 et 0.88 . 


\subsubsection{La satisfaction au travail}

La mesure de la satisfaction au travail est dérivée du questionnaire sur le vécu au travail (van Veldhoven \& Meijman, 1994) dans lequel le «Short Inventory to Monitor Psychosocial Hazard » est mobilisé (Notelaers, De Witte, van Veldhoven \& Vermunt, 2007). Cinq items sont utilisés pour évaluer la satisfaction au travail : «Je fais ce travail parce qu'il faut bien, cela veut tout dire »; «En général, j’aime entamer la journée de travail»; «Je trouve mon travail toujours aussi passionnant »; «J'éprouve du plaisir dans mon travail »; « Je dois à chaque fois vaincre ma propre résistance au travail ». L'échelle de mesure employée est «oui » ou «non». La consistance interne de l'outil de mesure est satisfaisante (Alpha de Cronbach de 0.81). La contribution factorielle des items varie entre 0.86 et 0.95 .

\subsubsection{L'implication organisationnelle}

L'échelle de mesure utilisée pour évaluer l'implication est adaptée des travaux de van Veldhoven \& Meijman (1994) par le premier auteur. Cinq items sont mis en avant pour évaluer l'implication organisationnelle : "J'estime que mes opinions correspondent largement à celles de l'organisation »; "J'estime important de pouvoir contribuer à la mission de cette organisation"; "Cette organisation me tient véritablement à cœur »; «Je me sens parfaitement à l'aise dans l'organisation»; «Le travail dans cette organisation est fort attrayant comparé à d'autres emplois que je pourrais obtenir ». Ces items mettent en avant les dimensions fondamentales de l'implication définie par d'autres auteurs tels que l'attachement affectif, le choix raisonné et l'obligation morale (Meyer \& Allen, 1997 ; Durrieu \& Roussel, 2002).

L'échelle de mesure employée est « oui » ou «non ». La consistance interne de l'instrument de mesure est satisfaisante (Alpha de Cronbach de 0.73). Les contributions factorielles des items mesurant l'implication organisationnelle varient de 0.78 à 0.88 .

\subsubsection{L'intention de départ de l'entreprise}

L'intention de départ est mesurée par les 4 items du questionnaire sur le vécu au travail (van Veldhoven \& Meijman, 1994) : " J'envisage parfois de changer d'emploi»; «J'envisage parfois de chercher du travail en dehors de cette organisation»; «J'ai l'intention de changer d'emploi l'année prochaine »; "L'année prochaine, j'ai l'intention de chercher un emploi en dehors de cette organisation ». L'échelle de mesure employée est «oui» ou « non ». La consistance interne de l'outil de mesure est satisfaisante (Alpha de 
Cronbach de 0.77). Toutes les contributions factorielles des items mesurant l'intention de départ sont très fortes.

\subsection{Les analyses de données}

Les hypothèses présentées dans le modèle (Figure 1) sont testées en utilisant l'approche confirmatoire des modèles d'équations structurelles à variables latentes, avec LISREL 8.88 (Joreskog, Sörbom, Du Toit \& Du Toit, 1999). Comme les indicateurs du harcèlement psychologique sont des variables ordinales, nous avons basé les analyses dans LISREL à partir des corrélations polysériales et polychoriques. Du fait de l'anormalité des distributions des variables, nous avons aussi utilisé la matrice de covariance asymptotique qui peut corriger les distributions qui ne sont pas multi-normales. Ensuite, toutes les estimations ont été calculées en utilisant la méthode d'estimation des moindres carrés (Joreskog \& Sorböm, 2003). Pour tester le modèle, nous avons suivi l'approche confirmatoire mise en avant par Joreskog \& Sorböm pour démontrer comment les hypothèses du modèle sont bien ajustées aux données. Divers indices d'ajustement sont retenus. D'abord des indices de mesure absolus ont été sélectionnés. Ils permettent d'évaluer dans quelle mesure le modèle théorique posé a priori reproduit correctement les données collectées (Roussel, 2005, p 272). La grande taille de l'échantillon $(n=6175)$ nous a fait choisir des indices peu sensibles à la taille de l'échantillon tels que le Goodness of Fit Index (GFI) et le Root Mean Square Error of Approximation (RMSEA). Ensuite des indices de mesure « incrémentaux » ou indices relatifs de comparaison ont été choisi. Ils évaluent l'amélioration de l'ajustement d'un modèle en le comparant à un modèle plus restrictif, dit « modèle de base ». Le Comparative Fit Index (CFI) et le Non-Normed Fit Index (NNFI) ont été choisis. Enfin, des indices de mesure de parcimonie ont été choisis. Ils permettent de déterminer, parmi plusieurs modèles plausibles équivalents, celui qui présente la meilleure parcimonie et qui devrait par conséquent, être préféré aux autres (Roussel, 2005, p273). Le Corrected Aiken Information Criterion (CAIC) est ici utilisé.

\section{Résultats}

Nous constatons d'abord que le modèle hypothétique est significatif avec un chi-deux de 2124.20 et 225 degrés de liberté. Le modèle apparaît donc bien ajusté selon la stratégie proposée par Joreskog : le RMSEA est avec 0.037 plus bas que 0.05. De plus, les autres indices démontrent un modèle satisfaisant. En effet, le GFI de 0.99, le NNFI de 0.98, le CFI de 0.99 et le CAIC de 2620.14, comparés au CAIC du modèle saturé de 2683.93 et le CAIC du modèle indépendant de 129844,68 indiquent un ajustement très satisfaisant du modèle. 
Nous constatons ensuite que toutes les hypothèses présentées dans le modèle sont confirmées (Figure 2). Toutefois, la première hypothèse - L'exposition à des comportements de harcèlement psychologique est négativement corrélée à la satisfaction au travail de la personne exposée - est celle qui reçoit la plus forte validation (0.49). Cette forte corrélation entre l'exposition au harcèlement et la satisfaction au travail confirme ainsi les travaux de Djurkovic, McCormack et Casimir (2004). La Figure 2 montre aussi que l'effet direct de l'exposition au harcèlement psychologique sur l'implication au travail (0.06) et l'effet direct du harcèlement sur l'intention de départ (0.08) sont moins importants que l'effet direct de l'exposition du harcèlement sur la satisfaction au travail (0.49). De plus, le poids de l'effet indirect de l'exposition au harcèlement est supérieur à l'effet direct. L'effet indirect de l'exposition au harcèlement et de la satisfaction au travail sur l'intention de départ est de 0.16 , et l'effet indirect de l'exposition au harcèlement, de la satisfaction au travail et de l'implication organisationnelle sur l'intention de départ est de 0.12. Les deux effets indirects excédent donc l'effet direct de l'exposition au harcèlement sur l'intention de départ. Si l'on compare l'effet global de l'exposition au harcèlement avec l'effet global de la satisfaction au travail sur la variable « intention de départ », l'effet global de la satisfaction au travail (0.56) excède l'effet global de l'exposition au harcèlement (0.38). La décision de départ de l'entreprise est par conséquent plus influencée par une baisse de la satisfaction au travail engendrée par l'exposition au harcèlement que par le harcèlement lui-même.

Ainsi les effets de l'exposition au harcèlement sur les réponses de l'individu ne sont pas identiques. D'abord, la satisfaction au travail est détériorée par l'exposition au harcèlement, ensuite l'implication au travail est affectée, enfin l'intention de quitter l'entreprise s'exprime (flèches en gras, figure 2). 
Figure 2 : Test des hypothèses par LISREL

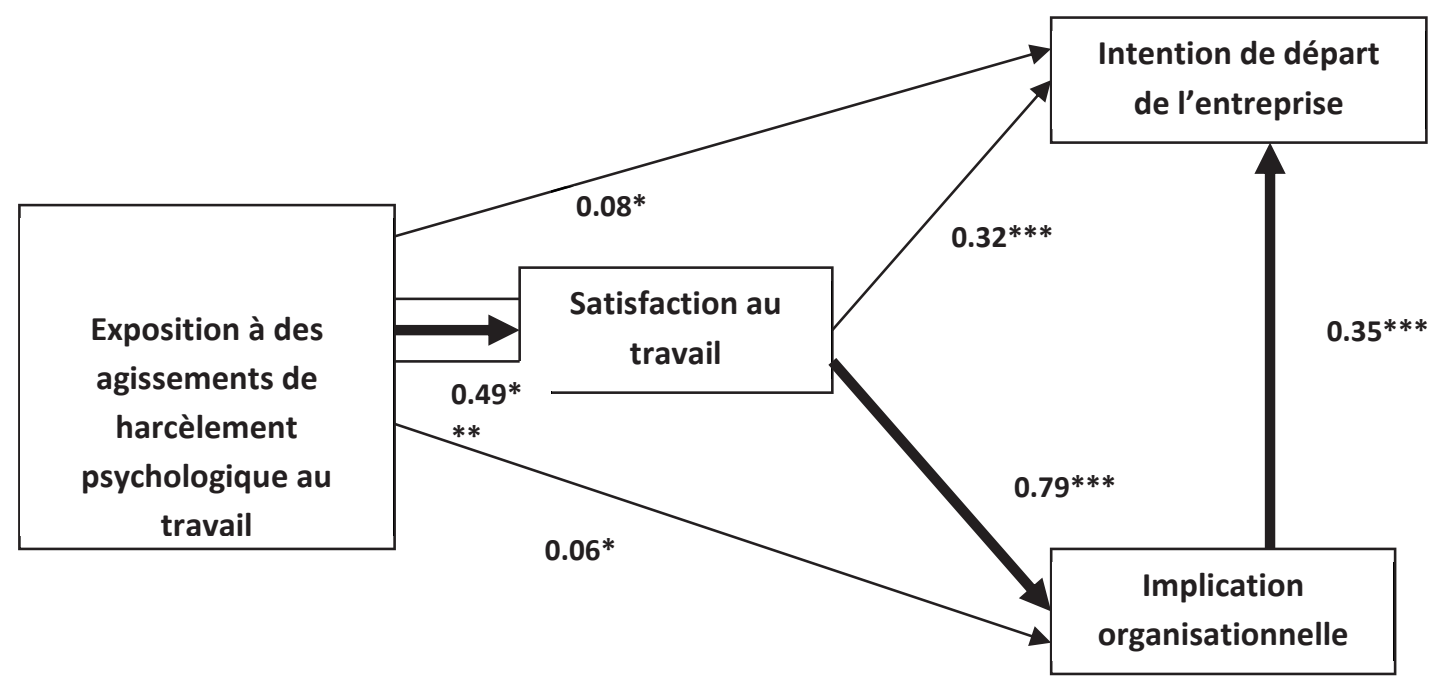

\section{Discussion et implications managériales}

Les limites de cette recherche sont nombreuses. D'abord, l'absence de représentativité de l'échantillon est notée. Cet échantillon n'est pas représentatif de la population active belge du fait d'une surreprésentation des agents de maîtrise. Les résultats de cette étude ne sont donc pas transposables à l'ensemble de la population active belge. Ensuite, les échelles de mesure choisies résultent des travaux réalisés par des chercheurs néerlandais. Si la fiabilité de ces outils a été très largement testée, leur faible notoriété en France n'amène pas naturellement l'adhésion du lecteur. Toutefois, ce questionnaire a été crée en 1994 et est depuis utilisé pour analyser le stress et promouvoir le bien-être au travail. Il est aujourd'hui devenu l'outil de mesure standard en Hollande et en Belgique et a été mobilisé près de 300000 fois dans des recherches réalisées au sein de ces deux pays. Enfin, l'étude se fonde sur un seul temps de mesure ce qui engendre un fort biais de variance de méthode commune. Toutefois, une seconde étude est aujourd'hui en cours avec un nouveau recueil de données.

Malgré ces limites, les résultats de la recherche sont d'un intérêt réel car toutes les hypothèses du modèle proposé sont validées. Deux apports semblent particulièrement essentiels.

Le premier apport est de révéler un effet direct de l'exposition à des comportements de harcèlement psychologique sur la satisfaction au travail (H1), l'implication organisationnelle $(\mathrm{H} 2 \mathrm{a})$ et l'intention de départ (H3a) de la personne exposée. Ce résultat confirme alors les recherches précédentes qui 
mettent en lien une telle situation de travail et les réponses émotionnelles et comportementales des individus (Bowling et Beehr, 2006; Djurkovic, McCormack et Casimir, 2004 ; Paolillo, Haubold, Leproust, D'escatha, Soula et Ameille, 2006 ${ }^{3}$; Quine, 1999). Dans une logique contractuelle de la relation de travail, le harcèlement psychologique apparaît donc comme un déterminant du déséquilibre du contrat psychologique relationnel. En effet, la perception de ne pas être respecté en situation de travail crée un déséquilibre dans la relation d'échange entre l'individu et l'organisation. Ce déséquilibre engendre alors deux types de réponse, l'une émotionnelle (par la baisse de satisfaction au travail) et l'autre comportementale: un «ajustement» comportemental de l'individu vise à rééquilibrer le contrat psychologique par une limitation partielle (baisse de l'implication) ou totale (départ de la personne) de sa contribution. Les réponses de l'individu illustrent ainsi son refus formulé du non respect de ses obligations par l'entreprise (non respect du contrat psychologique par le développement de comportements de harcèlement). Le concept de justice organisationnelle (Adams 1965 ; Greenberg 1987) paraît aussi intéressant pour expliquer l'impact de l'exposition au harcèlement psychologique sur la satisfaction, l'implication et l'intention de départ de l'entreprise. Le fait d'être exposé à des agissements de harcèlement développe une perception d'injustice chez l'individu qui le conduit à un état d'insatisfaction, à un désengagement et à un souhait de quitter l'entreprise. La mobilisation du concept de justice organisationnelle semble une piste de recherche intéressante à étudier dans des recherches futures.

Ce premier résultat met en avant que le harcèlement psychologique engendre des coûts considérables dans l'organisation. Une baisse de productivité liée à l'insatisfaction et au désengagement de l'individu, une perte de compétences liée au départ de la personne, des frais liés à un éventuel recrutement et à l'adaptation nécessaire de la personne à son poste et à l'organisation... constituent des coûts qui nuisent à la performance de l'entreprise. A ces coûts directs peuvent s'ajouter des coûts indirects liés notamment aux stratégies d'ajustement des témoins du harcèlement. Sans être exposé directement au harcèlement, le salarié témoin de telles pratiques peut en effet modifier sa représentation des obligations perçues par chacune des parties dans la relation d'échange et faire évoluer ses comportements en conséquence. Ainsi diverses études s'intéressent aux réactions des témoins de comportements de harcèlement (Desrumaux-Zagrodnicki, Lemoine, \& Mahon, 2004; Desrumaux, \& De Chacus, 2007 ; Desrumaux, Di Fabio, \& Przygodzki-Lionet,

\footnotetext{
${ }^{3}$ Cette étude menée entre le $1^{\text {er }}$ janvier 2000 et le 31 décembre 2002 auprès de 202 patients adressés à la consultation «souffrance au travail» de l'hôpital Raymond Poincaré de Garches montre que dans $65 \%$ des cas les personnes harcelées ont quitté l'entreprise: licenciement dans $62 \%$ des cas (avec $31 \%$ pour inaptitude, $11 \%$ après transaction, $20 \%$ pour faute, raison économique et désorganisation du service), retraite, démission et fin de CDD.
} 
2011). Elles offrent des pistes de recherche intéressantes et démontrent l'existence de coûts indirects du harcèlement psychologique au travail liés aux réactions des témoins de harcèlement.

L'existence de coûts directs et indirects liés aux réponses des personnes exposées et des témoins de harcèlement psychologique montre alors l'enjeu financier réel que représente le respect du contrat psychologique au sein de l'entreprise par la prévention des risques de harcèlement au travail. Tout l'intérêt de l'entreprise est alors de mettre en place des procédures de prévention afin d'éviter la remise en cause du contrat psychologique et l'émergence des coûts qui y sont liés. Ainsi, au-delà d'une logique sociale et sociétale qui tend à vouloir créer des lieux de travail respectueux des personnes, les logiques économique et financière sont autant d'arguments pour encourager et justifier des politiques de prévention du harcèlement psychologique au sein des organisations. Un véritable processus d'intervention proposé dans une recherche précédente (Poilpot-Rocaboy \& Winter, 2008, 2007) peut être mobilisé pour aller dans ce sens.

Le second apport de cette recherche est de révéler le rôle médiateur de la satisfaction au travail. En effet, la relation directe mise en avant s'accompagne d'une relation indirecte encore plus influente. L'exposition au harcèlement psychologique développe des affects négatifs forts chez l'individu qui se caractérisent par une baisse de sa satisfaction au travail. Puis, cet état émotionnel négatif conduit à une baisse de l'implication de l'individu dans l'organisation ( $\mathrm{H} 2 \mathrm{~b})$ et à l'intention de quitter l'entreprise (H3b). L'influence de la baisse de satisfaction au travail sur l'intention de départ (H3b) est plus faible que l'influence de la baisse de satisfaction au travail sur l'implication organisationnelle $(\mathrm{H} 2 \mathrm{~b})$ mais ensuite la baisse d'implication organisationnelle influence de manière importante l'intention de départ de l'entreprise (H4). Cette domination de l'influence indirecte par rapport à une influence directe du harcèlement psychologique est un résultat confirmé par d'autres recherches. Par exemple, McCormack, Casimir, Djurkovic et Yang (2006) montrent que l'impact de la satisfaction au travail sur l'implication organisationnelle est très fort et dépasse l'influence directe du harcèlement sur l'implication des personnes.

Le rôle médiateur de la satisfaction au travail dans la relation entre le harcèlement psychologique, l'implication organisationnelle et l'intention de départ est donc repéré. Ce résultat confirme les travaux réalisés par Hulin et Judge (2003), et Crede et al. (2007) sur le rôle médiateur de la satisfaction dans la relation entre une situation de travail en général et les réponses comportementales des individus (dont l'implication organisationnelle et l'intention de départ).

Ainsi, les résultats de cette étude montre l'existence d'un processus de réponse dominant des personnes exposées au harcèlement psychologique au 
sein des entreprises. Ce processus met en exergue trois étapes dont la première est une réponse émotionnelle qui conduit à l'insatisfaction au travail. Une seconde étape est la traduction de ces émotions négatives en actions de désimplication organisationnelle puis d'intention de départ (troisième étape) de la personne exposée (voir flèches en gras dans la figure 2).

Ce processus de réponse émotionnel dans un premier temps puis comportemental dans un second temps, met en avant le rôle des émotions pour expliquer les comportements des individus au sein des organisations. Cette thématique du rôle et de l'importance des émotions au travail apparait alors une piste de recherche future d'un grand intérêt. La "Affective Events Theory" (AET) semble un cadre théorique possible pour continuer dans ce sens. Weiss et Cropanzano (1996) suivi par d'autres auteurs (Desrumaux, Di Fabio, \& Przygodzki-Lionet 2011; Desrumaux, Ntsame-Sima, \& Leroy 2011) s'intéressent aux émotions et au lien entre émotions, harcèlement, performance et comportements au travail.

Face à ce processus de réponse dominant des personnes exposées au harcèlement psychologique, l'enjeu pour l'entreprise devient alors de «bloquer» le processus de réponse individuelle de la personne exposée au harcèlement psychologique à la première étape (émergence d'émotions négatives). Dans une logique économique et financière, ce «blocage » permettrait d'éviter l'émergence des coûts directs et indirects repérés précédemment.

Le soutien social (Bruchon-Schweitzer, 2002 ; Ruiller, 2008). semble un concept à mobiliser pour limiter l'émergence d'émotions négatives et assurer le blocage du processus de réponse à son étape première. Les trois formes de soutien social - instrumental, informatif et émotionnel- semblent pouvoir être mobilisées dans le cadre du harcèlement psychologique. Toutefois, l'analyse du type de harcèlement est une étape préalable nécessaire. En effet, le choix du «bon » soutien social semble en lien avec le type de harcèlement vécu. Selon les situations (harcèlement vertical, horizontal ou stratégique), nous pensons que l'efficacité du «blocage » du processus par le soutien social sera différente.

Dans le cas d'un harcèlement psychologique stratégique où le harcèlement apparaît comme une politique d'entreprise (exemple: souhait d'éviter les coûts de licenciement d'une personne en la poussant à la démission...), nous pensons que les soutiens possibles sont le soutien informatif (information sur les droits de la personne en lien avec le code du travail...) et le soutien émotionnel développé par les collègues ou les instances représentatives du personnel. Nous restons cependant peu optimistes quand à la capacité du soutien social interne à l'entreprise à bloquer le processus de réponses individuelles à sa phase première. Un soutien externe, familial, amical, médical, associatif et surtout légal (plainte de la victime) ainsi que le départ de la 
personne peuvent être mobilisés dans une logique de protection de la santé physique et mentale de la personne.

Dans le cas d'un harcèlement vertical (harcèlement par un responsable hiérarchique direct...), le soutien instrumental -par un changement de poste décidé par la DRH ou la Direction Générale, par une sanction du harceleur...-, le soutien informatif -par le rappel des valeurs et des comportements attendus dans l'organisation par la DRH et la Direction Générale (comportement respectueux, absence de violence...)-, et le soutien émotionnel (écoute, empathie, manifestation de confiance...) de la DRH et de la Direction Générale, sont des moyens utiles pour bloquer le processus de réponses. Ces trois formes de soutien semblent en effet en mesure de limiter le développement d'émotions négatives et d'empêcher la mise en action de la personne exposée (désengagement et intention de départ).

Enfin, dans le cas d'un harcèlement horizontal (harcèlement par un collègue ou collaborateur), le soutien instrumental, le soutien informatif et le soutien émotionnel notamment du manager semblent également à même de bloquer le processus de réponse individuelle à la première étape. Le rôle du manager par sa compréhension des émotions négatives de l'individu, par son écoute, par ses décisions de sanction du harceleur... semble ici déterminant.

Cette recherche met en exergue une double influence directe et indirecte de l'exposition au harcèlement psychologique sur la satisfaction au travail, l'implication organisationnelle et l'intention de départ du salarié. Elle met en exergue la dominance de l'influence indirecte sur l'influence directe et fait émerger l'impact émotionnel fort du harcèlement et ses conséquences sur les comportements individuels et les coûts organisationnels. Ainsi, le harcèlement psychologique apparaît comme un dysfonctionnement coûteux tant pour l'individu que pour l'organisation qui conduit à justifier le développement de politiques de prévention et à mobiliser le concept de soutien social pour tenter de bloquer le processus de réponse de l'individu exposé.

\section{Références}

Adams, J. S. (1965). Inequity in Social Exchange in Berkowitz L. (ed), Advances in Experimental Social psychology, New York, Academic Press, 2, 267-299.

Ayoko, O. B., Callan, V. J., \& Hartel, C. E. J. (2003). Workplace conflict, bullying, and counterproductive behaviours. International Journal of Organizational Analysis, 11, 4, 283-301.

Bowling, N. A., \& Beehr, T. A. (2006). Workplace Bullying From the Victim's Perspective: A Theoretical Model and Meta-Analysis. Journal of Applied Psychology, 91, 5, 998-1012.

Bruchon-Schweitzer, M. (2002). Psychologie de la santé. Modèles, concepts et méthodes. Paris, Dunod. 
Crede, M., Chernyshenko, O. S., Stark, S., Dahal R. S., \& Bashshur M. (2007). Job Satisfaction as mediator: An assessment of job satisfaction's position within the nomological network. Journal of Occupational and Organizational Psychology, 80, 515-538.

Desrumaux, P. (2007). Harcèlement moral au travail, survictimation et problèmes du harceleur : quand les victimes sont jugées aussi responsables que leurs harceleurs. Cahiers Internationaux de Psychologie Sociale, 73, 61-73.

Desrumaux, P., \& De Chacus, S. (2007). Bullying at Work : Effects of the Victim's Pro and Antisocial Behaviors and of the Harassed's Overvictimization on the Judgments of Help-giving. Studia Psychologica,49(4), 357-368.

Desrumaux, P., Di Fabio, A-M., \& Przygodzki-Lionet, N. (2011). Harcèlement moral au travail : effet de la gravité des agissements, de leur fréquence, du statut du harceleur et de la sur-victimisation du harcelé sur les jugements de responsabilité, les émotions et les intentions d'aide, Counseling.

Desrumaux, P., Ntsame-Sima, M \& Leroy, N. (2011). Harcèlement moral au travail et pro/antisocialité du salarié victime : quels effets sur les jugements, les émotions et les intentions d'aide des témoins. Psychologie du Travail et des Organisations.

Desrumaux-Zagrodnicki, P., Lemoine, C., \& Mahon, P. (2004). Harcèlement moral et climats d'entreprise : effets de facteurs humains et organisationnels sur les jugements d'équité. Psychologie du Travail et des Organisations, 10, 29-44.

Depolo, M., Guglielmi, D., \& Toderi, S. (2004). Prévenir le harcèlement moral au travail : le rôle du contrat psychologique. Psychologie du Travail et des Organisations, 10, 88-100.

Djurkovic, N., McCormack, D., \& Casimir, G. (2004). The physical and psychological effects of workplace bullying and their relationship to intention to leave: a test of the psychosomatic and disability hypotheses, International Journal of Organizational Theory and Behavior, 7, 4, 469-497.

Durrieu, F., \& Roussel, P. (2002). L'implication organisationnelle dans les réseaux de franchises: un concept pertinent pour des entreprises en réseau ?, Revue de Gestion des Ressources Humaines, 44, 2-19.

Einarsen, S. (1999). The nature and causes of bullying at work. International Journal of Manpower, 20, 1, 0143-7720.

Einarsen, S., Hoel, H., Zapf, D., \& Cooper, C. (2003). Bullying and emotional abuse in the workplace. International perspectives in research and pratice. London and NewYork : Taylor and Francis.

Einarsen, S., Raknes, B. I., \& Matthiesen, S. B. (1994). Bullying and harassment at work and their relationships to work environment quality : an exploratory study. European Work and Organizational Psychologist, 4, 4, 381-401.

Einarsen, S., Hoel, H., \& Notelaers, G. (2009). Measuring exposure to bullying and harassment at work:Validity, factor structure and psychometric properties of the Negative Acts Questionnaire-Revised. Work \& Stress, 23, 1, 24-44.

Einarsen, S. E., \& Nielsen, M. B. (2004). 4th International Conference on Bullying and Harassment in the Workplace, Bergen, Norway.

Einarsen, S. E., \& Mikkelsen, E. G. (2003). Individual effects of exposure to bullying at work, in S. Einarsen, H. Hoel, D. Zapf, C.L. Cooper (Eds.), Bullying and Emotional Abuse in the workplace. International perspectives in research and practice (127144). London: Taylor \& Francis. 
Einarsen, S., \& Hoel, H. (2001). The Negative Acts Questionnaire: development, validation and revision of a measure of bullying at work, Paper presented at the 10th European Congress on Work and Organizational Psychology, Prague, May.

Einarsen, S.E., \& Raknes, B. (1997). Harassment in the workplace and victimization of men. Violence and Victims, 12, 247-263.

Einarsen, S., \& Skogstad, A. (1996). Bullying at work : epidemiological findings in public and private organizations. European Journal of Work and Organizational Psychology, 10 (4), 185-201.

Einarsen, S. E. (1996). Bullying and harassment at work: Epidemiological and psychosocial aspects, University of Bergen, Bergen.

Eriksen, W., \& Einarsen, S. (2004). Gender minority as a risk factor of exposure to bullying at work : the case of male assistant nurses. European Journal of Work and Organizational Psychology, 13(4), 473-492.

Faulx, D. (2009). Les deux faces de la construction sociale du harcèlement moral Nouveaux regards et nouvelles réalités dans le monde du travail, Psychologie du Travail et des Organisations, 15, 1, 5-20.

Greenberg, J. (1987). A taxonomy of organizational justice theories, Academy of Management Review, 12, 1, 9-22.

Griffeth, R. W., Hom, P. W., \& Gaertner, S. (2000). A meta-analysis of antecedents and correlates of employee turnover: update, moderator tests and research implications for the next millennium, Journal of Management, 26, 463-488.

Hauge, L. J., Skogstad, A., \& Einarsen, S. (2007). Relationships between work environment quality and bullying: Results of a representative study, Work \& Stress, $21,3,220-242$.

Hodson, R., Roscigno, V. J., \& Lopez, S. H. (2006). Chaos and the Abuse of power: Workplace Bullying in Organizational and Interactional Context, Work and Occupations, 33, 382-416.

Hoel, H., Einarsen, S. E, \& Cooper C. L. (2003). Organisational effects of bullying, in S. Einarsen, H. Hoel, D. Zapf \& C.L. Cooper (Eds.), Bullying and Emotional Abuse in the workplace. International perspectives in research and practice (145-161). London: Taylor \& Francis

Hoel, H., \& Salin, D. (2003). Organisational antecedents of workplace bullying, in S. Einarsen, H. Hoel, D. Zapf \& C.L. Cooper (Eds.), Bullying and Emotional Abuse in the workplace. International perspectives in research and practice (203-218). London: Taylor \& Francis.

Hulin, C. L., \& Judge, T. A. (2003). Job attitudes, in W.C. Borman, D.R. IIgen, R.J. Klimoski (Eds.), Handbook of Psychology, 12, 255-276. Hoboken: Wiley.

Jöreskog, K., \& Sörbom, D. (2003). LISREL 8.54. Programm. SSI Central.

Jöreskog, K. (2005). Structural Equation Modeling with Ordinal Variables using LISREL. Karl's Corner: www.ssicentral.com.

Joreskog, K., Sörbom, D., Du Toit, S., \& Du Toit, M. (1999). Lisrel 8 : New Statistical Features. Chicago, Scientific Software International.

Lambert E. G., Hogan N. L., \& Barton S. M. (2001). The impact of job satisfaction on turnover intent: a test of a structural measurement model using a national sample of workers, The Social Science Journal, 38, 233-250.

Levinson H., Price C., Munden K., Mandl H., \& Solley C. (1962). Men, management and mental health, Cambridge, M.A. Harvard University Press.

Leymann, H. (1996). The content and development of mobbing at work. European Journal of Work and Organizational Psychology, 5, 165-184. 
Leymann, H. (1990a). Mobbing and Psychological terror at workplaces, Violence and Victims, 5, 119-126 ; (1990b). Manual of the LIPT questionnaire for assessing of the risk of psychological violence at work. Stockholm. Violen.

Locke, E. A. (1976). The Native and causes of Job Satisfaction, in M.D. Dunnett (ed) Handbook of Industrial and organizational Psychology, Chicago : Rond Mc Nally, 1297-1349.

Maertz, C. P., \& Campion, M. A., (1998). 25 years of voluntary turnover research: a review and critique. In C.L. Cooper \& I.T. Robertson (Eds), International Review of Industrial and Organizational Psychology, (49-86). Toronto : John Wiley \& Sons Ltd.

Matthiesen, S. B., \& Einarsen, S. (2004). Psychiatric distress and symptoms of PTSD among victims of bullying, British Journal of Guidance \& Counselling, 32, 3, 335 356.

McCarthy, P., Sheehan, M., \& Kearns, D. (1995). Managerial Styles and their Effect on Employees Health and Well-Being in Organizations Undergoing Restructuring. Report for Worksafe Australia: Griffith University, Brisbane.

McCarthy, P., Rylance, R., Bennett, R., \& Zimmerman, H. (Eds.) (2001). Bullying: From backyard to boardroom. Sydney: Federation Press.

McCarthy, P., Sheehan, M., Wilkie, S., \& Wilkie, W. (Eds.) (1998). Bullying: causes, costs and cures. Brisbane: Beyond Bullying Association.

McCormack, D., Casimir, G., Djurkovic N., \& Yang L. (2006). The concurrent effects of workplace bullying, satisfaction with supervisor, and satisfaction with co-workers on affective commitment among schoolteachers in China, International Journal of Conflict Management, 17, 4, 316-331.

Meyer, J., \& Allen, N. (1997). Commitment in the Workplace: Theory, Research and Application. Sage Publications, Thousand Oaks, CA.

Meyer, J. P., Stanley, D. J., Herscovitch, L., \& Topolnytsky, L. (2002). Affective, continuance and normative commitment to the organization: A meta-analysis of antecedents, correlates, and consequences, Journal of Vocational Behavior, 61, 2052.

Mowday, R. T. (1998). Reflections on the study and relevance of organizational commitment. Human Resource Management Review, 8, 4, 387-401

Mowday, R. T., Porter, L. W., \& Steers R. M., (1982). Employee-Organization : the psychology of commitment, absenteeism and turnover. Ed Academic Press, New York.

Mueller, C. W., Wallace, J. E., \& Price, J. L., (1992). Employee commitment, Work and Occupations, 19, 211-236.

Nielsen, M. (2009). Methodological issues in research on workplace bullying. Operationalisations, measurements, and samples. Unpublished Phddissertation.Unpublished manuscript, University of Bergen, Norway.

Nielsen, M. B., Mattiesen, S. B., \& Einarsen, S. (2004). When the bully is a leader: The relationship between destructive leaders and symptoms of posttraumatic stress disorder among victims of workplace bullying. Paper presented to the Fourth International Conference on bullying and harassment in the Workplace, Bergen, 125-127.

Notelaers, G., De Witte, H., \& Einarsen, S. (2010). A job characteristics approach to explain workplace bullying. European Journal of Work and Organizational Psychology. 19, 4, 487-504. 
Notelaers, G., \& Einarsen, S. (2008). The Construction and Validity of the Short Negative Acts Questionnaire. Paper presented at the 6th International Conference on Workplace Bullying: Sharing your Knowlegde, Montreal, Canada.

Notelaers, G., De Witte, H., van Veldhoven, M., \& Vermunt, J. K. (2007). Construction and validation of the Short Inventory to Monitor Psychosocial Hazards. Médecine du Travail \& Ergonomie, 44, 11-17.

Notelaers, G., Einarsen, S., De Witte, H., \& Vermunt, J.K. (2006). Measuring exposure to bullying at work: The validity and advantages of the latent class cluster approach. Work \& Stress, 20, 4, 288-301.

Paolillo, A. G., Haubold, B., Leproust, H., D'escatha, A., Soula, M.C., \& Ameille, J. (2006). Devenir socioprofessionnel de patients en situation de harcèlement moral présumé. Documents pour le médecin du travail, 105, 49-59.

Poilpot-Rocaboy, G., \& Winter R. (2008). Combattre le harcèlement psychologique au travail : Proposition d'un processus d'intervention, Gérer et Comprendre, 94, 38-49.

Poilpot-Rocaboy, G., \& Winter R. (2007). Combating Psychological Harassment in the Workplace: Processes for Management Intervention. International Employment Relations Review, 13, 1, 67-83.

Poilpot-Rocaboy, G. (2006). Bullying in the Workplace: A Proposed Model for Understanding the Psychological Harassment Process. Research and Practice in Human Resource Management, 14, 2, 1-17.

Poilpot-Rocaboy, G. (1998). Violation du contrat psychologique et harcèlement professionnel. Gestion 2000, 4, 15-34.

Quine, L. (1999). Workplace bullying in NHS community trust: staff questionnaire survey, British Medical Journal, 318, 228-232.

Rayner, C., \& Cooper, C.L. (1997). Workplace Bullying: Myth or reality- Can We afford to ignore it?, Leadership and Organization Development Journal, 18, 211214.

Roussel, P. (2005). Méthodes de développement d'échelles pour questionnaires d'enquête, in P. Roussel et F. Wacheux Management des Ressources Humaines. Méthode de recherche en Sciences Humaines et Sociales (245-348). De Boeck, Bruxelles.

Ruiller, C. (2008). Le soutien social au travail : conceptualisation, mesure et influence sur l'épuisement professionnel et l'implication organisationnelle : l'étude d'un cas hospitalier. Thèse de doctorat de Sciences de Gestion.

Schein E. H. (1965). Organizational Psychology. Englewood Cliffs, NJ : Prentice Hall.

Tett, R. P., \& Meyer, J. P. (1993). Job satisfaction, organizational commitment, turnover intention, and turnover: Path analysis based on meta-analytic finding. Personnel Psychology, 46, 259-293.

van Veldhoven, \& M., Meijman, T.F. (1994). Vragenlijst Beleving en Beoordeling van de Arbeid, NIA. Amsterdam.

Weiss, H. M., \& Cropanzano, R. (1996). An affective events approach to job satisfaction. In B.M. Staw \& L.L. Cummings (eds), Research in organizational behaviour, 18, 1-74, Greenwich, CT : JAI Press.

Zapf, D., \& Einarsen, S. (2003). Individual antecedents of bullying, in S. Einarsen, H. Hoel, D. Zapf \& C.L. Cooper (Eds.), Bullying and Emotional Abuse in the workplace. International perspectives in research and practice (165-184). London: Taylor \& Francis.

Zapf, D. (1999). Organizational work group related and personal causes of mobbing/bullying at work. International Journal of Manpower, 20, 1 et 2, 70-85. 
G. Poilpot-Rocaboy, G. Notelaers \& L. Johan Hauge / Psychologie du Travail et des Organisations 21(2015) 358-379

Zapf, D., \& Gross, C. (2001). Conflict escalation and coping with workplace bullying: a replication and extension. European Journal of Work and Organization Psychology, $10,4,497-522$. 